\title{
AN ADAPTIVE OBSERVER FOR ON-LINE TOOL WEAR ESTIMATION IN TURNING, PART II: RESULTS
}

\author{
K o u o s h Danal and A. Galip Ulsoy \\ Department of Mechanical Engineering and Applied Mechanics, University of Michigan, Ann Arbor, \\ Michigan 48109-2125, U.S.A.
}

(Received March 1986, and in revised form July 1986)

\begin{abstract}
The basic concept and design of an adaptive observer for tool wear estimation in turning, based on force measurement, has been presented in the previous paper (Part I). This paper shows that numerical problems in the estimation of the states of tool wear precludes the use of this method in multi-wear cases where both flank wear and crater wear are present. The method can be applied, however, when one type of wear (either flank wear or crater wear) dominates. The method is applied in turning experiments to a case where flank wear is dominant, and to a second case where crater wear dominates. For the first case the flank wear estimates show excellent agreement with actual wear measurements. For the second case the crater wear estimates are satisfactory, but not as good as in the first case.
\end{abstract}

\section{INTRODUCTION}

In Part I an adaptive observer has been proposed for on-line tool wear estimation. The design of this adaptive observer relies on a dynamic state model of tool wear developed for this purpose. In this model, which is formed using the existing analytical and/or empirical relationships available in the literature, the state variables represent abrasive flank wear, diffusive flank wear and crater wear. The input is the feed, and the output is the cutting force.

Before the adaptive observer is designed, the model's suitability for parameter and state estimation is evaluated. For this evaluation the model's general behavior is studied by simulation. The simulation results show good agreement with typical results published in the manufacturing literature [1]. Since the linear model is to be used as the basis for parameter and state estimation, its stability, controllability, and observability conditions are also analysed. In order to analyse these conditions, the model, which is highly non-linear, is linearised in several regions of its trajectory. The linear model is found to be unstable, as expected, due to the increasing nature of the wear process. It is also controllable with the input (feed), and observable with the output (cutting force). Next, the adaptive observer is designed and tuned for the non-linear model, and shows very good performance when results are compared with the actual values from the non-linear simulation.

In this paper the development of the adaptive observer for tool wear estimation is continued with the reconstruction of the wear components from the observed states. It is shown in the paper that this reconstruction requires the periodic measurement of tool wear. Therefore, a certain amount of off-line testing may be required for this purpose. However, before the implementation of the adaptive observer and the collection of experimental results, the feasibility of the reconstruction process should be investigated. For this purpose, the exact transformation matrix relating the observed states and the 
wear components is obtained from the linearised model, and used to reconstruct the wear components from the observed states obtained in simulation.

These simulation results indicate that the reconstructed states are not accurate. The inaccuracy of the reconstructed states, which is due to the approximate nature of the estimated states, and the high level of precision required for this transformation, show that when both types of wear are present, it is not feasible in practice to separate their effect by this method.

The analysis of the linearised model also indicates that the model parameters show large variations due to excessive wear. This means that it should be possible to detect tool failure by parameter estimation alone. To check the performance of the adaptive observer in a single-wear case, and also investigate the behavior of the estimated mode1 parameters during tool failure, turning experiments are conducted. The set-up is composed of a CNC lathe, a laboratory microcomputer, a three component force dynamometer, an analog-to-digital converter, an instrumentation tape recorder, and an analog filter. Two sets of experiments are performed (one causing flank wear and the other causing crater wear as the single dominant wear component) and the cutting force data is recorded. The adaptive observer is then tuned and applied to the recorded data.

The estimated flank wear from the first test shows very good agreement with the actual values of tool wear measured at several points during the cut. The estimated crater wear from the second test is also compared with the measured crater wear and the results are satisfactory, but not as good as the flank wear estimates in the first test. The estimated parameters show high sensitivity to disruptions in the cutting process (e.g. chip entanglement or "hard spots"), as well as to tool failure. This indicates that the estimated model parameters can be used as an indication of deviations from a normal cutting operation,

\section{RECONSTRUCTION OF THE WEAR COMPONENTS}

The reconstruction of the wear components from the observed states requires a transformation component. As shown in Appendix A, this transformation component (a matrix in this case) can only be obtained when both forms (i.e. the original form and the observer form) of the linear mode1 are known. Since the cutting process is treated as a "black box", the original form of the linear model is not available. Therefore, a certain amount of off-line testing is required to estimate this transformation matrix. To illustrate this point, let us consider a simple example.

\subsection{EXAM PLE}

Assume, for simplicity, that only one kind of tool wear is present. A linear model representing the instantaneous behavior of such a process has the form

$$
\begin{aligned}
& \dot{x}=a^{\prime} x+b^{\prime} u \\
& y=c^{\prime} x+d^{\prime} u
\end{aligned}
$$

where: $\mathrm{x}$ is the wear component, $\boldsymbol{u}$ represents the input and $\boldsymbol{y}$ is the output and a', $\mathbf{b}^{\prime}, \mathbf{c}^{\prime}$ and $\mathbf{d}^{\prime}$, the model coefficients, all depend on the process variables such as the cutting conditions and the tool-workpiece combination. In order to make the above model suitable for digital computation, the above continuous-time linear model is transformed into discrete-time form by using a zero-order hold and a constant sampling period. The discrete-time form of the above mode1 has the form

$$
\begin{gathered}
x(k+1)=p^{\prime} x(k)+q^{\prime} u(k) \\
y(k)=c^{\prime} x(k)+d^{\prime} u(k) .
\end{gathered}
$$



form

In order to design the adaptive observer the above model is transformed into observer

$$
\begin{gathered}
x_{0}(k+1)=-\mathrm{ax},(\mathrm{k})+b u(k) \\
y(k)=x_{0}(k)+d^{\prime} u(k)
\end{gathered}
$$

such that

$$
x=T^{\prime} x_{0}
$$

where

$$
T^{\prime}=\frac{1}{c^{\prime}}
$$

The observer then, will have the form,

$$
\hat{x}_{0}=-\hat{a} \hat{x}_{0}+\hat{b} u(k)+g\left[y(k)-\left(\hat{x}_{0}+\hat{d}^{\prime}\right) u(k)\right]
$$

where: $\hat{a}, \hat{b}, \hat{d}^{\prime}$, and $\hat{x}_{\mathbf{0}}$ are the estimates of a, $\boldsymbol{b}, \boldsymbol{d}$, and $\boldsymbol{x}_{\mathbf{0}}$ in equations (5) and (6) respectively, and $\mathrm{g}$ is the observer gain.

Note that the estimated state, $\hat{x}_{\mathbf{0}}$, will have to be transformed into an estimate of tool wear, $\hat{\boldsymbol{x}}$ (the state of the original model), to be meaningful. For this transformation we need to know $\boldsymbol{T}^{\prime}$ in equation (7). For this simple case, however, $\boldsymbol{T}^{\prime}$ is the inverse of the parameter c' (see equation (8)) which defines the relationship between the output and the state variable in the original linear model (see equation (2)). Since the original linear model, as discussed earlier, is not known (the cutting process is treated as a "black box"), the parameter c' is not known. This means that the transformation component $T^{\prime}$ is not available, and the estimated state is not reconstructible.

However, the parameter c', which represents the effect of wear on the output, can be estimated by off-line testing. This implies that the reconstruction of the wear component from the estimated state is possible through a transformation component which in this simple case can be obtained by the off-line estimation of a single parameter.

The reconstruction of the wear components from the observed states in the case of higher order models, however, depends on a transformation matrix which is much more difficult to obtain than the scalar transformation parameter in the above example. In cases where different types of tool wear are present, the order of the model representing the wear process increases (e.g. the order is three for the mode1 presented in Part I). This higher order of the model causes the transformation matrix to be much more involved algebraically, such that, the transformation matrix cannot be obtained by the off-line estimation of a single parameter (as was the case in the example).

Of course, in practice, the transformation between the wear components and the observed states does not necessarily have to be defined through a transformation matrix. Such a transformation can be defined as

$$
\hat{x}=f\left(\hat{x}_{0}\right)
$$

where $\hat{x}_{0}$ are the estimated states from the adaptive observer. In order to determine $f\left(\hat{x}_{0}\right)$, real values of tool wear, $\boldsymbol{x}$, will be determined in different intervals during the cut (by off-line measurement), and are related to the observed states (from the adaptive observer) by regression analysis. Of course, the form of the relationship $f\left(\hat{x}_{0}\right)$ and the regression technique should be selected carefully. Once $f\left(\hat{x}_{0}\right)$ has been determined equation (10) can be used to reconstruct the wear components. 
Before a regression based method as described above is utilised, however, the accuracy of the reconstruction process should be investigated. For this purpose, an exact transformation matrix, obtained from the non-linear model (see Appendix A for the computation method used), is used to reconstruct the wear components.

In order to check the accuracy of the transformation process, the relationship

$$
x=T x_{0}
$$

was used to transform the observed states in Part I into the states of the original form of the model. These results are shown in Table 1, and indicate that the reconstructed states are not reliable even with the exact transformation matrix $T$. In fact, the mean and standard deviation of these results (also shown in Table 1) indicate that the reconstructed states are far from being acceptable.

Every component of the adaptive observer-the parameter estimator, and state observer-were shown to provide accurate results (the errors in the observed states are less than $\pm 5 \%$ (see Part I) and expecting a higher accuracy in the estimated states is not realistic). The error involved in reconstructing the wear components is particularly due to the magnitudes of the states in the original form (the wear components are numerically very small). The effect of this constraint is particularly noticeable in the errors associated with $\hat{x}_{2}$ and $\hat{x}_{3}$ which require much higher accuracy (see Table 1).

The results in Table 1 and the preceeding analysis prove that the reconstruction of the wear components from the observed states in the third order mode1 presented in Part I, although theoretically possible, is not practical. However, in cases where only one type of wear is present (as in the Example above), the reconstruction of the wear components relies on only one transformation component and numerical sensitivity is not an issue.

T ABLE 1

Reconstructed states using $\mathrm{T}$ and $\hat{\boldsymbol{x}}_{0}$

\begin{tabular}{ccccccc}
\hline $\begin{array}{c}\text { Case } \\
\text { no. }\end{array}$ & \multicolumn{1}{c}{$\hat{\boldsymbol{x}}_{\mathbf{1}}$} & $\hat{\boldsymbol{x}}_{2}$ & $\dot{\boldsymbol{x}}_{3}$ & $\boldsymbol{e}_{1}$ & $\boldsymbol{e}_{2}$ & $\boldsymbol{e}_{3}$ \\
\hline $\mathbf{1}$ & $\mathbf{0 \cdot 2 2 6 6}$ & -155.56 & $-\mathbf{7 7 \cdot 8 3 4}$ & -0.1002 & 155.56 & $77 \cdot 834$ \\
2 & 0.04430 & -92.48 & -46.289 & 0.0960 & 92.4813 & 46.289 \\
3 & 0.04591 & -83.445 & $-\mathbf{4 1 \cdot 7 7 2}$ & 0.0963 & 83.448 & 41.772 \\
4 & 0.04657 & -77.99 & -39.05 & 0.0962 & 78.00 & 39.0486 \\
5 & $0-04786$ & -72.339 & -36.22 & 0.0955 & 72.345 & 36.2 \\
6 & $\mathbf{0 . 0 8 3 5 3}$ & -18.496 & -9.2655 & 0.0342 & 18.50 & 9.267 \\
$\mathbf{7}$ & $\mathbf{0 . 0 9 1 9 4}$ & -13445 & -6.740 & 0.0298 & 13.457 & 6.7440 \\
8 & 0.09621 & -9.7968 & -4.9167 & 0.0264 & $9 \cdot 8205$ & 4.9234 \\
9 & $\mathbf{0 \cdot 1 0 5 5}$ & -5.9733 & -3.0070 & 0.0186 & 6.0154 & 3.0170 \\
10 & 0.1333 & -1.9163 & -0.9829 & -0.0057 & 2.0012 & 0.9977 \\
11 & 0.1285 & -6.3127 & -4.7182 & -0.0160 & 6.3195 & 4.7276 \\
12 & 0.1315 & -3.9765 & -2.9651 & -0.0162 & 3.9995 & 2.9874 \\
13 & 0.1498 & -1.6747 & -1.2338 & -0.0336 & 1.7270 & 1.2700 \\
14 & 0.8288 & 0.6881 & 0.9621 & -0.7070 & -0.5002 & -0.9040 \\
15 & 0.1389 & -0.3543 & -0.2658 & -0.0476 & 0.3593 & 0.3117 \\
16 & 0.1398 & 0.1703 & 0.2755 & -0.0377 & -0.0566 & -0.1943 \\
17 & $\mathbf{0 \cdot 1 2 3 0}$ & 0.1594 & 0.2629 & -0.0196 & -0.1335 & -0.1531 \\
18 & $\mathbf{0 \cdot 1 1 5 4}$ & 0.1661 & 0.2684 & -0.0122 & -0.1296 & -0.1420 \\
19 & 0.1183 & 0.2632 & 0.3698 & -0.0155 & -0.2042 & -0.2200 \\
20 & $\mathbf{0 \cdot 1 4 7 1}$ & 0.2845 & 0.4005 & -0.0438 & -0.1803 & -0.2248 \\
Mean & & & & -0.0281 & 27.1415 & $\mathbf{1 3 \cdot 6 7 8 6}$ \\
S.D. & & & & 0.1689 & 44.1405 & 22.0526 \\
\hline
\end{tabular}


It was decided to experimentally evaluate the adaptive observer for such cases, since they are of practical interest.

From the simulation results in Part I the parameters of the non-linear model change drastically when significant amounts of wear develop. This change in parameters has also been reported in $[2,3]$ where tool failure is detected by observing sudden changes in the parameters. It was decided to also investigate the feasibility of tool failure detection through the parameter estimates.

\section{THE EXPERIMENTAL SET-UP AND RESULTS}

The objective of the designed experiment was to implement the adaptive observer to (i) estimate tool wear in cases where only one type of wear is dominant, and (ii) detect tool failure and/or breakage by observing sudden changes in the estimated parameters. The implementation of the adaptive observer was based on cutting force as the output signal, therefore, a force transducer was needed. The measured cutting force would be affected by measurement and process noise, therefore, an analog filter was needed to separate the signal from the noise. The adaptive observer was implemented digitally, therefore, a microcomputer capable of data aquisition was needed. The schematic diagram of the experimental set-up is presented in Fig. 1. This scheme would be suitable for the ultimate implementation of the adaptive observer. However, since cutting tests are both time consuming and expensive in terms of workpiece material and tools, it was decided to record the cutting force data using an instrumentation tape recorder to avoid exhaustive repetition of the tests for the purpose of tuning and evaluating the adaptive observer.

\subsection{EXPERIMEN T}

One of the requirements for the tests was to have only one type of wear present for each cut. In order to satisfy this requirement several tests were run to identify the proper cutting conditions. Two sets of cutting conditions were determined (see Table 2). One set produced flank wear (cut no. 1) and the other produced crater wear (cut no. 2) as

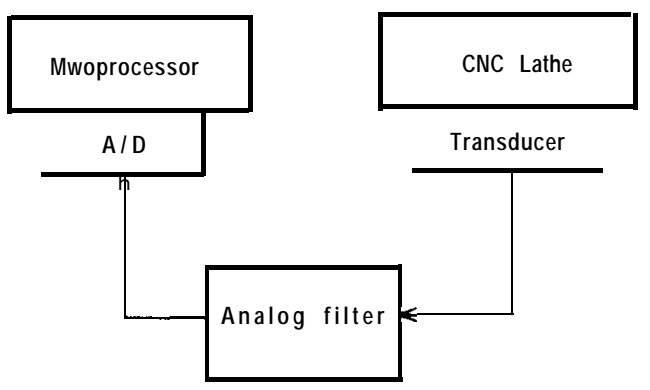

Figure 1. Schematic of the experimental set-up.

TABLE 2

The cutting conditions used for cuts nos. 1 and 2

\begin{tabular}{cccccc}
\hline $\begin{array}{c}\text { Cut } \\
\text { no. }\end{array}$ & $\begin{array}{c}\boldsymbol{v} \\
(\mathrm{m} / \mathrm{min})\end{array}$ & $\begin{array}{c}\boldsymbol{f} \\
(\mathrm{mm} / \mathrm{rev})\end{array}$ & $\begin{array}{c}\mathbf{d} \\
(\mathrm{mm})\end{array}$ & $\begin{array}{c}\text { Tool } \\
\text { material }\end{array}$ & $\begin{array}{c}\text { Workpiece } \\
\text { material }\end{array}$ \\
\hline 1 & 366 & 0.025 & $1 \cdot 27$ & $\begin{array}{c}\text { Carboloy TNG 432 } \\
\text { Carboloy TNG 432 }\end{array}$ & $\begin{array}{c}\text { Ann'd 4340 } \\
\text { Ann'd 4340 }\end{array}$ \\
\hline
\end{tabular}


the major tool wear. Also, in order to investigate the effect of tool failure on the parameters cut no. 1 was continued until the tool broke. All three components of the cutting force were recorded for each cut. The recorded data were then used to tune the adaptive observer. Tuning the adaptive observer required the determination of:

\subsubsection{The appropriate component of the cuttingforce}

It is generally believed that all the components of the cutting force are affected by wear. However, the level of this effect on the different components of the cutting force is not known. In order to select the proper component, all three components were plotted and their relationship to the measured wear were visually noted (see Figs. 2 and 3). Although all three components of the cutting force were significantly affected by wear, the radial component of cutting force showed a much more pronounced effect due to tool breakage in cut no. 1. Therefore, it was decided to use the radial component of the cutting force as the output signal.

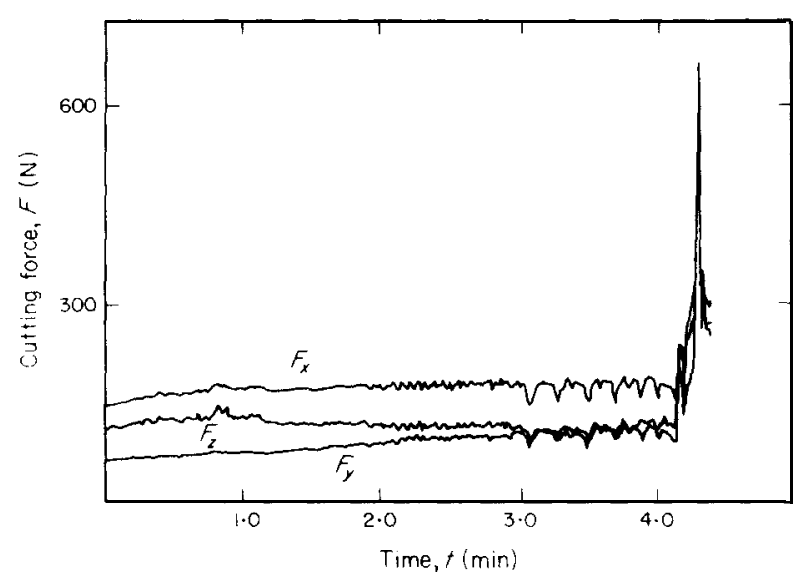

Figure 2. The three components of the cutting force in cut no. I. $F_{x}$, normal component; $F_{v}$, radial component; $F_{z}$, feeding component.

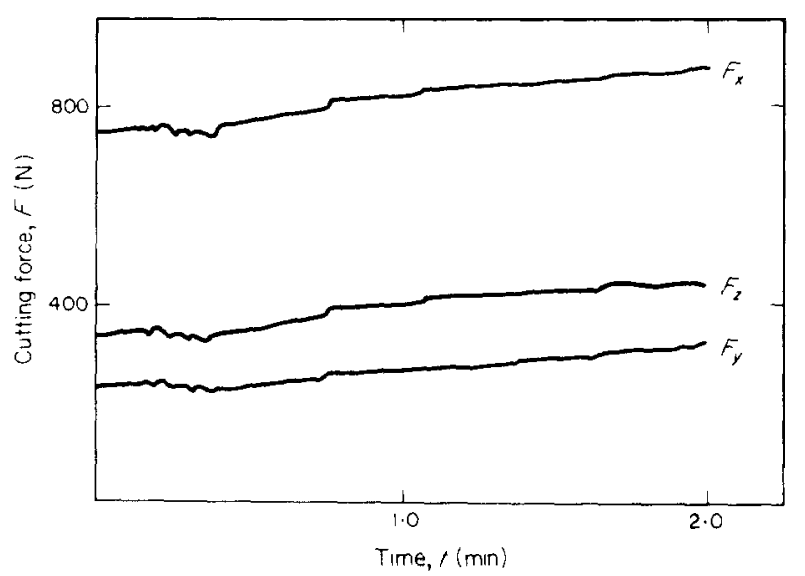

Figure 3. The three components of the cutting force in cut no. 2. $F_{x}$, normal component; $F_{v}$, radial component; $F=$, feeding component. 


\subsubsection{The sampling period}

Wear is a slow process, and its response time is on the order of minutes [1]. Therefore, a sampling period on the order of minutes is quite adequate to characterise the effect of wear on the output. On the other hand, a second objective of the test was to detect tool failure through the detection of sudden changes in the estimated parameters. Therefore, fast detection of tool failure was also a criterion in the determination of the sampling period. It was decided to use a sampling period of $1 \mathrm{sec}$ for data acquisition. This sampling period was short enough to represent the effect of wear on the cutting force while providing relatively quick detection of tool failure. The selected sampling period resulted in a sampling frequency of $1 \mathrm{~Hz}$.

\subsubsection{The filtering frequency}

The measured cutting force contained a fair amount of measurement and process noise. In cases where the noise frequency and the useful frequency content of the signal are non-overlapping, noise must be filtered from the signal to prevent aliasing [4]. In order to determine the frequency of the noise, the frequency spectra of the signal was analysed using a spectrum analyser. Figure 4 shows the frequency spectrum of the radial cutting force in cut no. 1. From this figure one can observe that the spectrum of this signal is quite "flat" except at $4.5 \mathrm{kHz}$ where a peak occurs. The occurrence of this peak is due to the lateral vibration of the workpiece in the absence of a tailstock on the lathe. Since the frequency range of interest for the purpose of wear estimation was between 0 and $0.05 \mathrm{~Hz}[1]$, and the frequency of the lateral vibration of the workpiece was significantly far from the useful signal, the vibration frequency could be filtered from the signal. According to the Sampling Theorem the filter cutoff frequency must be at most half the sampling frequency to prevent aliasing [4]. Therefore, in our case a filter frequency of $0.5 \mathrm{~Hz}$ was sufficient to prevent aliasing. Although we could select a filtering frequency lower than $0.5 \mathrm{~Hz}$, in order to avoid unnecessary lags caused by a lower filtering frequency, $0.5 \mathrm{~Hz}$ was chosen.

\subsubsection{The voltage range on the analog-to-digital converter (ADC) board}

The ADC board used for data acquisition had a 12 bit converter. This meant that the digital range provided for computation was 0 to 4095 (or -2048 to 2048). The ADC board

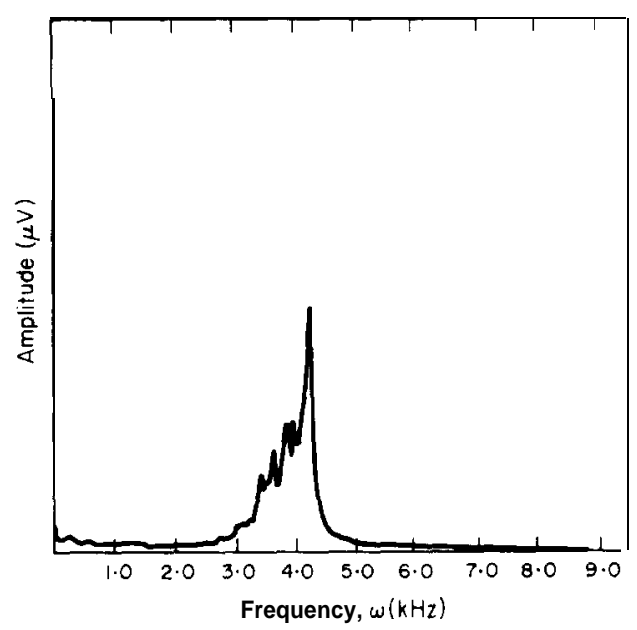

Figure 4. Typical frequency spectrum of the cutting force data from the tests. 
was also software adjustable for different analog input levels. Therefore, in order to use the full range of the converter (maximise the resolution and minimise the quantisation error) it was essential to use an input level which matched the measured voltage level from the dynamometer. Since the output of the tape recorder was restricted to $1 \mathrm{~V}$, in order to minimise the quantisation error, the input range on the converter was adjusted to the smallest range of $\pm 1 \cdot 28 \mathrm{~V}$. Also, in order to use the maximum resolution further, amplifiers were used to amplify the signal to the upper limit of the input range used in the ADC. The selected input range on the converter provided a resolution of $0.31 \mathrm{mV}$ which for a maximum cutting force of about $1000 \mathrm{~N}$ would be approximately $0.25 \mathrm{~N}$.

\subsubsection{The type of parameter estimation algorithm}

As with the output of any real process, the measured signals are not completely noise free, and parameter estimators are known to show bias in the estimates in the presence of noise. Among parameter estimation algorithms, however, the ones based on the output error method are known to be less affected by noise [5]. It was decided to use the output error method for parameter estimation. Such an algorithm is explained in detail in Appendix B. For this particular problem the estimator parameters were selected as

$$
\begin{gathered}
\mathbf{P}(0)=\left[\begin{array}{cc}
1 & 0 \\
0 & 1000
\end{array}\right] \\
\beta=0.95
\end{gathered}
$$

and

$$
D\left(q^{-1}\right)=1-0 \cdot 5 q^{-1}
$$

\subsubsection{Transformation component}

The reconstruction of the wear components requires a transformation component. As discussed in the example, this transformation component (1/c) for a first order system, represents the effect of wear on the cutting force, and must be estimated by off-line testing. This off-line testing requires the measurement of tool wear at several points during the cut so that the relationship between wear and cutting force can be determined. Tool wear measurements were obtained by a tool-makers microscope in the case of Bank wear and a proficorder in the case of crater wear. For flank wear, the average width of wear on the flank side of the tool was determined; and for crater wear, the maximum depth of the crater groove on the face of the tool was measured. The measured values of flank wear and crater wear are shown in Figs. 7 and 8 respectively. It was assumed that the relationship between wear and cutting force is linear, therefore, the estimated transformation component $(1 / \hat{c})$ is a constant. The values of $\hat{\boldsymbol{c}}$ were determined as the ratio between the change in the cutting force from the initial (sharp tool) value and the measured flank wear or crater wear. The average value of $\hat{\boldsymbol{c}}$ in each test was then used in the transformation component. The value of $\hat{\boldsymbol{c}}$ for cuts no. 1 and 2 were $108.78 \mathrm{~mm} / \mathrm{N}$ and $371.00 \mathrm{~mm} / \mathrm{N}$ respectively.

\subsection{DISCUSSION OF THE RESULTS}

The adaptive observer was applied to the recorded radial cutting force in cuts no. 1 and 2. The estimated parameters from the tests are shown in Figs. 5 and 6 respectively, and the estimated wear results are shown in Figs. 7 and 8 respectively. Based on these results one can conclude that: 


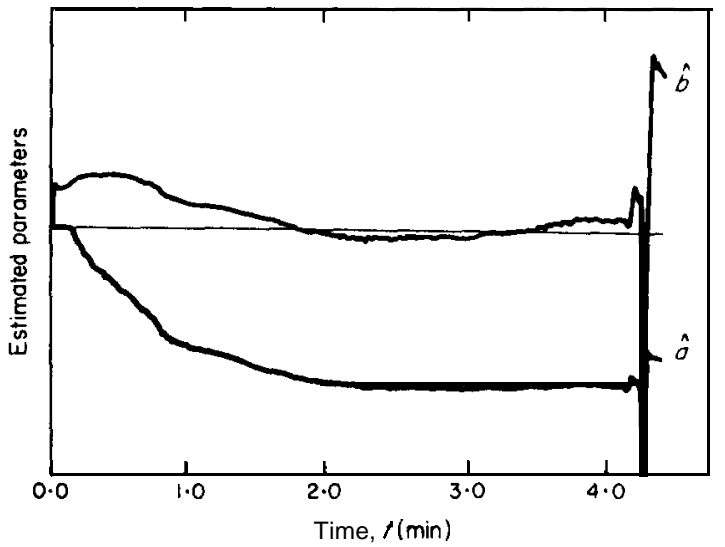

Figure 5. Estimated parameters in cut no. 1.

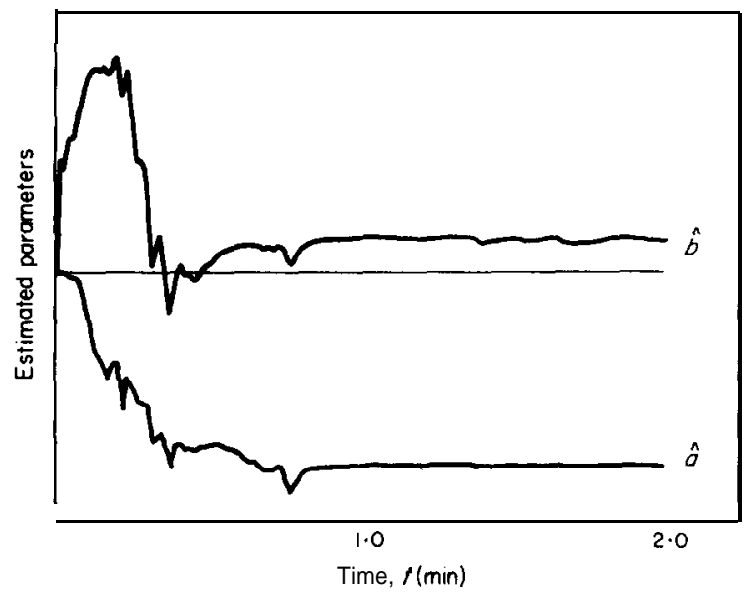

Figure 6. Estimated parameters in cut no. 2.

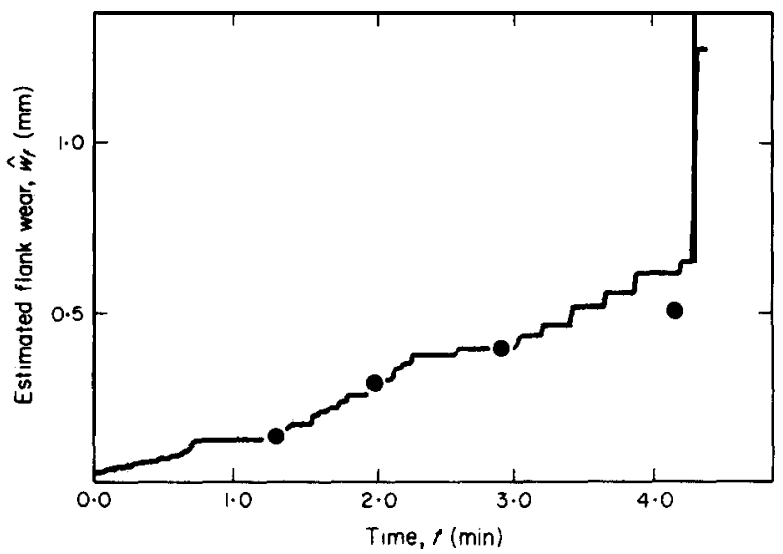

Figure 7. Estimated flank wear in cut no. 1. Measured values. 


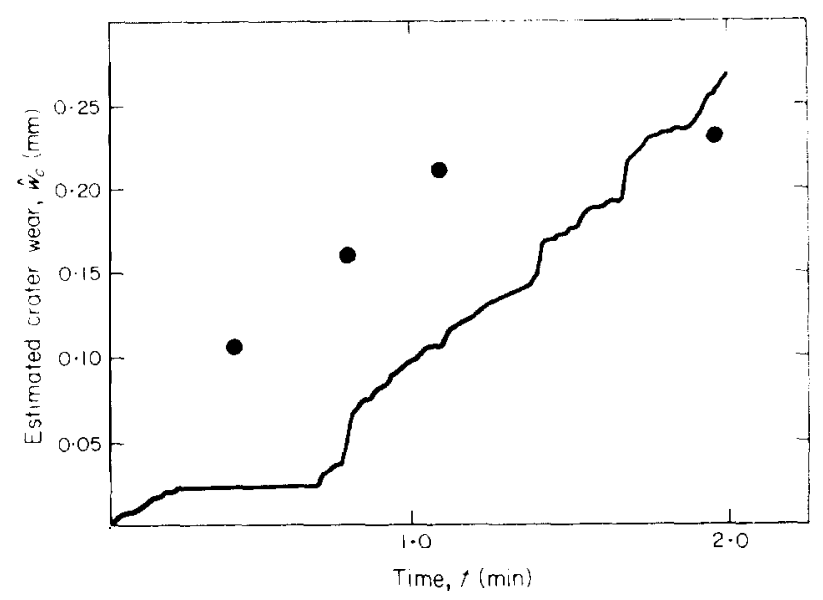

Figure 8. Estimated crater wear in cut no. 2. Measured values.

1. The estimated parameters do show a rather drastic change due to tool breakage (see Fig. 5). Therefore, the estimated parameters can be used for the purpose of tool failure detection.

2. The estimated flank wear in cut no. 1 was very close to the measured values (see Fig. 7), therefore, the assumption that the relationship between flank wear and cutting force in cut no. 1 was linear was indeed confirmed to be a useful one.

3. The estimated crater wear in cut no. 2 was not close to the measured values, except at the end of the cut (see Fig. 8). This means that the assumption that $\mathrm{c}$ in cut no. 2 is constant was not an accurate one.

The above approach for tool wear estimation is identical to the empirical approach discussed in Part I for constant inputs. However, in cases where the input is changed for control purposes, the adaptive observer is superior to the empirical approach. In such cases, the direct effect of the input on the cutting force is removed from the signal through the estimated component $D$, and the indirect effect of the input on the cutting force (through the wear components) is accounted for through the vector b (see Part I). The capability to separate the effect of the input through the model makes the approach also superior to tool failure detection methods such as $[2,3]$ in which the effect of input is not considered. Another advantage of the adaptive observer is that $\mathbf{a}, \mathbf{b}$, and $D$ are continually updated from process measurement, and only c must be determined from off -line testing.

Perhaps, the main drawback in using the adaptive observer for tool wear estimation is its dependence on off-line testing, and the tuning of the adaptive observer which requires the determination of several parameters (e.g. $\mathrm{g}$ in equation (9) and $\mathrm{P}, \boldsymbol{\beta}$, and $D$ in equations (12)-(14)). However, for large lot sizes and cases where only one type of wear is dominant, the use of the adaptive observer for the purpose of tool wear estimation and tool failure detection is very well justified.

The off-line estimation of the parameter $\mathrm{c}$ can also be performed by using computer vision systems currently under development for on-line tool wear estimation [6]. Such a combined measurement system takes advantage of the complementary features of the two methods. Intermittent tool wear measurements can be made using vision at the end of the cuts when the tool comes out of contact with the workpiece. These measurements can then be compared with the estimated wear by the adaptive observer to periodically update the parameter c. The adaptive observer then serves to track the developing tool 
wear during actual cutting, even under variable cutting conditions, while the vision system provides less frequent but direct wear measurements between parts.

\section{SUMMARY AND CONCLUSIONS}

The tool wear estimation process proposed involves the transformation of the observed states to the states of the original model, which must be established by off-line estimation. However, before the method is implemented, the accuracy of the transformation process should be investigated. For this, an exact transformation matrix obtained from the non-linear mode1 is used to reconstruct the observed states from the simulation. The results are found to be quite inaccurate, which implies that the required degree of accuracy imposed on the transformation due to the magnitude of the wear components make the method unreliable for multi-wear cases.

In order to check the feasibility of the model for on-line tool wear estimation in cases where only one type of wear is present, the designed adaptive observer has been implemented in a real cutting process. In these tests it was essential to restrict the tool wear to only one type, therefore, two sets of cutting conditions were selected for this purpose. One set produces flank wear and the other produces crater wear as the major type of tool wear. The adaptive observer was then implemented using the recorded cutting force data from these tests. In order to reconstruct the wear components, a transformation component is needed, and has been obtained by off-line wear measurements. The estimated results show very good agreement with the measured values of tool wear in the case of flank wear. In the case of crater wear, however, the estimated results are not as satisfactory, and this is due mainly to the non-linear relationship between cutting force and crater wear.

The following topics, as a result of the current study, may warrant further investigation:

1. Incorporation into an adaptive control system. One of the main advantages of the present approach is its suitability for incorporation into an adaptive control system. Especially since the method has been proven applicable for a single-wear case, and most turning cuts fit in this category.

2. Use of vision (between cuts) to track wear during cuts. As mentioned above, the main disadvantage of the method is its requirement for the off-line estimation of the transformation component. However, if some periodic means of automatic wear measurement is available, the transformation component can be determined and updated during the cut. Such wear measurement is possible using vision between cuts. Especially since the long processing time required by vision systems does not impose a restriction in this case.

3. Use of multi-input multi-output models. The design of the adaptive observer is based on a single-input single-output (SISO) model. The use of a SISO model, however, was primarily for simplicity. Therefore, the method can be designed based on a multi-input multi-output (MIMO) model. In such a MIMO model feed and cutting speed can be considered as the inputs and cutting force and temperature can be used as the outputs. In fact, whenever practical, the use of temperature as the output variable or one of the output variables may make the model more useful, and possibly resolve the short coming of the method in the multi-wear cases.

\section{REFEREN CES}

1. K. Danal and A. G. Uzsoy 1985 in Sensors and Controls for Manufacturing, pp. 137-148. A dynamic state model for on-line tool wear estimation in turning. American Society of Mechanical Engineers.

2. S. Takata, M. Ogawa, P. Bertok, J. Oоtsuka, K. Matushima and T. Sata 1985 Robotics and Computer-Integrated Manufacturing Vol. 2, pp. 33-40. Real-time monitoring of tool breakage using Kalman filtering. Oxford: Pergamon. 
3. M. S. LAN and Y. NAERHEIM 1985 in Sensors and Controls for Manufacturing, pp. 49-56. In-process detection of tool breakage in milling. American Society of Mechanical Engineers

4. K. J. Astrom and B. Wittenmark 1984 Computer Controlled Systems. Englewood Cliffs, NJ: Prentice-Hall.

5. B. D. 0. AnDerson 1985 A utomarica 21, 247-258. Adaptive systems, lack of persistency of excitation and bursting phenomena.

6. S. K. JetLy 1984 Proceedings of the North American Manufacturing Research Conference, pp. 255-259. A new radiometric method of measuring drill wear. Society of Manufacturing Engineers.

7. Y. таканashi, M. J. Rabins, and D. M. Auslander 1972 Control. Reading, MA: AddisonWesley.

8. G.C. Goopwis and K. S. Sin 1984 Adaptioe Filtering, Prediction, and Control. Englewood Cliffs, NJ: Prentice-Hall.

\section{APPENDIX A: COMPUTATIONAL METHOD}

For the purpose of the reconstruction of the wear components from the observed states the transformation matrix $\mathrm{T}$ is needed. This appendix discusses the methodology used to compute the transformation matrix $\mathrm{T}$.

In order to compute the transformation matrix $\mathrm{T}$, the third order SISO non-linear model developed in Part I must be linearised. This non-linear model has the general form

$$
\begin{aligned}
& \dot{\mathbf{x}}=\mathbf{f}^{\prime}(\mathbf{x}, u, t) \\
& y=g^{\prime}(\mathbf{x}, u, t)
\end{aligned}
$$

where: $\mathrm{x}$ is a third-order state vector, $\boldsymbol{u}$ is a scalar input and $\mathrm{y}$ is a scalar output.

The non-linear model can be linearised according to the linearisation method of Part I. Such a linear model has the form

$$
\begin{gathered}
\dot{\mathbf{x}}=\mathbf{A x}+\mathbf{b} u \\
y=\mathbf{c}^{T} \mathbf{x}+D u
\end{gathered}
$$

\section{DISCRETE-TIME FORM}

The continuous-time linear model defined in equations (A3) and (A4) can be transformed into discrete-time form using a zero-order hold and a constant sampling period

$$
\begin{gathered}
\mathbf{x}(k+1)=\operatorname{Px}(k)+\mathbf{q} u(k) \\
y(k)=\mathbf{c}^{T} \mathbf{x}(k)+D u(k)
\end{gathered}
$$

where:

$$
\begin{gathered}
\mathrm{P}=\exp (\mathbf{A} \Delta t) \\
\mathbf{q}=(\mathbf{P}-\mathbf{I}) \mathbf{A}^{-1} \mathbf{b}
\end{gathered}
$$

and $\Delta \boldsymbol{t}$ is the sampling period. For computational purposes, $\mathrm{P}$ and $\mathrm{q}$ in the above equations can be obtained by the power series expansion of the term exp (A $\Delta t)$ [7].

\section{CANONICAL FORM}

As discussed in Part I, in order to design the adaptive observer, the model should be transformed into observer form

$$
\begin{gathered}
\mathbf{x}_{0}(k+1)=\tilde{\mathbf{P}} \mathbf{x}_{0}(k)+\tilde{\mathbf{q}} u(k) \\
y(k)=\tilde{\mathbf{c}}^{T} \mathbf{x}_{0}(k)+D u(k) .
\end{gathered}
$$


The parameters of the model in canonical form can be obtained from the transfer function of the discrete-time model defined by equations (A5) and (A6).

$$
\frac{Y(z)}{U(z)}=\frac{b_{0}+\sum_{j=1}^{m} b_{j} z^{-j}}{1+\sum_{i=1}^{n} a_{i} z^{-i}}+D
$$

The above transfer function can be obtained from the relationship

$$
G(z)=\frac{Y(z)}{U(z)}=\mathbf{c}^{T}[z \mathbf{I}-\mathbf{P}]^{-1} \mathbf{q}+D .
$$

\section{THE TRANSFORMATION MATRIX}

The transformation matrix $\mathrm{T}$ in the relationship

$$
\mathbf{x}=\mathbf{T} \mathbf{x}_{\mathbf{0}}
$$

is required to reconstruct the wear components from the observed states. This transformation matrix $\mathrm{T}$ can be obtained through the observability matrices of the models in the original form and the observer form, such that

$$
\mathbf{T}=\boldsymbol{\Theta}^{-1} \tilde{\boldsymbol{\Theta}}
$$

where:

$$
\begin{aligned}
& \Theta=\left[\mathrm{c}, \mathbf{P}^{T} \mathbf{c},\left(\mathbf{P}^{T}\right)^{2} \mathbf{c}, \ldots,\left(\mathbf{P}^{T}\right)^{n-1} \mathbf{c}\right] \\
& \tilde{\Theta}=\left[\tilde{\mathbf{c}}, \tilde{\mathbf{P}}^{T} \tilde{\mathbf{c}},\left(\tilde{\mathbf{P}}^{T}\right)^{2} \tilde{\mathbf{c}}, \ldots,\left(\tilde{\mathbf{P}}^{T}\right)^{n-1} \tilde{\mathbf{c}}\right]
\end{aligned}
$$

In the above relationships, $\mathrm{P}$ and $\mathrm{c}$ are as in equations (A5) and (A6) and $\tilde{\mathbf{P}}$ and $\tilde{\mathbf{c}}$ are as in equation (A9) and (A10). Equation (A14) is used to compute, the transformation matrix $\mathrm{T}$.

\section{APPENDIX B: OUTPUT ERROR METHOD}

The parameter estimation algorithms based on the output error method have the general form [8]

$$
\begin{gathered}
\hat{\boldsymbol{\theta}}(k)=\hat{\boldsymbol{\theta}}(k-1)+\frac{\mathbf{P}(k-2) \overline{\boldsymbol{\phi}}(k-1)}{\beta+\overline{\boldsymbol{\phi}}(k-1)^{T} \mathbf{P}(k-2) \overline{\boldsymbol{\phi}}(k-1)} \bar{\nu}(k) \\
\mathbf{P}(k-1)=\frac{1}{\beta}\left[\mathbf{P}(k-2)-\frac{\mathbf{P}(k-2) \overline{\boldsymbol{\phi}}(k-1) \overline{\boldsymbol{\phi}}(k-1)^{T} \mathbf{P}(k-2)}{\beta+\overline{\boldsymbol{\phi}}(k-1)^{T} \mathbf{P}(k-2) \overline{\boldsymbol{\phi}}(k-1)}\right]
\end{gathered}
$$

where: the parameter $\mathrm{P}(\mathrm{k})$ is the $(\boldsymbol{n}+\mathrm{m}, \boldsymbol{n}+\boldsymbol{m})$ matrix of estimation gains, $\overline{\boldsymbol{\phi}}(\boldsymbol{k})$ is the vector of known variables, $\hat{\boldsymbol{\theta}}(\boldsymbol{k})$ is a vector of parameter estimates, and $\bar{\nu}(\boldsymbol{k})$ is the estimation error. In the above algorithm

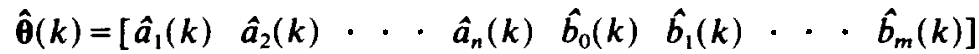

$$
\begin{aligned}
& \bar{\phi}(k)=[-\bar{y}(k-1)-\bar{y}(k-2) \cdots-\bar{y}(k-n) \quad \mathrm{u}(\mathrm{k}-1) \quad \mathrm{u}(\mathrm{k}-2) \quad \cdots u(k-m)]
\end{aligned}
$$


where:

$$
\begin{gathered}
\bar{y}(k)=\overline{\boldsymbol{\phi}}(k-1)^{T} \hat{\boldsymbol{\theta}}(k) \\
\hat{y}(k)=\overline{\boldsymbol{\phi}}(k-1)^{T} \hat{\boldsymbol{\theta}}(k-1)
\end{gathered}
$$

and the estimation error $\overline{\boldsymbol{\nu}}(\boldsymbol{k})$ is defined as

$$
\bar{\nu}(k)=y(k)-\hat{y}(k)+\left[D\left(q^{-1}\right)-1\right][y(k)-\bar{y}(k)] .
$$

$D\left(q^{-1}\right)$ in the above equation is a fixed moving average filter defined as

$$
D\left(q^{-1}\right)=1+d_{1} q^{-1}+\cdots+d_{1} q^{-1} .
$$

